\title{
Erratum to: A new measure of divergence with its application to multi-criteria decision making under fuzzy environment
}

\author{
Rajkumar Verma $^{1} \cdot$ Shikha Maheshwari ${ }^{2}$
}

Published online: 7 October 2016

(C) The Natural Computing Applications Forum 2016

\section{Erratum to: Neural Comput \& Applic}

\section{DOI 10.1007/s00521-016-2311-y}

The original article has been published online with some errors. The errors are corrected with this erratum.

1. In Eq. (12) and in all other places, where the factor $\frac{1}{\sqrt{e-1}}$ occurs, it is in error. The correct factor is $\frac{1}{\sqrt{e}-1}$.

2. Just before Eq. (18), there is in error. It has '-' sign. The correction is for the sign to be ' + '.

3. Equation (20) should be:

The online version of the original article can be found under doi:10.1007/s00521-016-2311-y.

Rajkumar Verma

rkver83@gmail.com

Shikha Maheshwari

maheshwari.shikha23@gmail.com

1 Department of Applied Sciences, HMR Institute of Technology and Management, Guru Gobind Singh Indraprastha University, Hamidpur, Delhi 110036, India

2 Department of Mathematics, Jaypee Institute of Information Technology, Noida, Uttar Pradesh 201307, India 


$$
\begin{gathered}
=\frac{1}{n(\sqrt{e}-1)}\left[\sum_{x_{j} \in X_{1}}\left\{\begin{array}{c}
\left\{\begin{array}{c}
\left(\frac{\mu_{A}\left(x_{j}\right)+\mu_{B}\left(x_{j}\right)}{2}\right) \exp \left(\frac{2-\mu_{A}\left(x_{j}\right)-\mu_{B}\left(x_{j}\right)}{2}\right) \\
+\left(\frac{2-\mu_{A}\left(x_{j}\right)-\mu_{B}\left(x_{j}\right)}{2}\right) \exp \left(\frac{\mu_{A}\left(x_{j}\right)+\mu_{B}\left(x_{j}\right)}{2}\right)
\end{array}\right\} \\
-\left\{\begin{array}{c}
\left(\mu_{A}\left(x_{j}\right) \exp \left(1-\mu_{A}\left(x_{j}\right)\right)+\left(1-\mu_{A}\left(x_{j}\right)\right) \exp \left(\mu_{A}\left(x_{j}\right)\right)\right) \\
+\left(\mu_{B}\left(x_{j}\right) \exp \left(1-\mu_{B}\left(x_{j}\right)\right)+\left(1-\mu_{B}\left(x_{j}\right)\right) \exp \left(\mu_{B}\left(x_{j}\right)\right)\right)
\end{array}\right.
\end{array}\right\}\right) \\
+\sum_{x_{j} \in X_{2}}\left\{\begin{array}{c}
+\left(\frac{2-\mu_{A}\left(x_{j}\right)-\mu_{A}\left(x_{j}\right)}{2}\right) \exp \left(\frac{\mu_{A}\left(x_{j}\right)+\mu_{A}\left(x_{j}\right)}{2}\right) \\
2
\end{array}\right\} \\
\left.-\left\{\begin{array}{c}
\left(\mu_{A}\left(x_{j}\right) \exp \left(1-\mu_{A}\left(x_{j}\right)\right)+\left(1-\mu_{A}\left(x_{j}\right)\right) \exp \left(\mu_{A}\left(x_{j}\right)\right)\right) \\
+\left(\mu_{A}\left(x_{j}\right) \exp \left(1-\mu_{A}\left(x_{j}\right)\right)+\left(1-\mu_{A}\left(x_{j}\right)\right) \exp \left(\mu_{A}\left(x_{j}\right)\right)\right) \\
2
\end{array}\right\}\right) .
\end{gathered}
$$

4. The expression following Eq. (21) is in error, the correct form is as follows:

$$
\begin{aligned}
& \left.=\frac{1}{n(\sqrt{e}-1)} \sum_{j=1}^{n}\left[\begin{array}{c}
{\left[\begin{array}{c}
\left(\frac{\mu_{A}\left(x_{j}\right)+1-\mu_{B}\left(x_{j}\right)}{2}\right) \exp \left(\frac{1-\mu_{A}\left(x_{j}\right)+\mu_{B}\left(x_{j}\right)}{2}\right) \\
+\left(\frac{1-\mu_{A}\left(x_{j}\right)+\mu_{B}\left(x_{j}\right)}{2}\right) \exp \left(\frac{\mu_{A}\left(x_{j}\right)+1-\mu_{B}\left(x_{j}\right)}{2}\right)
\end{array}\right\}} \\
-\left\{\begin{array}{c}
\left(\mu_{A}\left(x_{j}\right) \exp \left(1-\mu_{A}\left(x_{j}\right)\right)+\left(1-\mu_{A}\left(x_{j}\right)\right) \exp \left(\mu_{A}\left(x_{j}\right)\right)\right) \\
+\left(\left(\mu_{B}\left(x_{j}\right)\right) \exp \left(1-\mu_{B}\left(x_{j}\right)\right)+\left(1-\mu_{B}\left(x_{j}\right)\right) \exp \left(\mu_{B}\left(x_{j}\right)\right)\right)
\end{array}\right.
\end{array}\right\}\right] \\
& \left.-\left[\begin{array}{c}
\left\{\begin{array}{c}
\left(\frac{1-\mu_{A}\left(x_{j}\right)+\mu_{B}\left(x_{j}\right)}{2}\right) \exp \left(\frac{1+\mu_{A}\left(x_{j}\right)-\mu_{B}\left(x_{j}\right)}{2}\right) \\
+\left(\frac{1+\mu_{A}\left(x_{j}\right)-\mu_{B}\left(x_{j}\right)}{2}\right) \exp \left(\frac{1-\mu_{A}\left(x_{j}\right)+\mu_{B}\left(x_{j}\right)}{2}\right)
\end{array}\right\} \\
-\left\{\frac{\left(\left(1-\mu_{A}\left(x_{j}\right)\right) \exp \left(\mu_{A}\left(x_{j}\right)\right)+\mu_{A}\left(x_{j}\right) \exp \left(1-\mu_{A}\left(x_{j}\right)\right)\right)}{2}\right.
\end{array}\right]\right] .
\end{aligned}
$$

5. The expression following Eq. (22) is in error, the correct form is as follows:

$$
\begin{aligned}
& =\frac{1}{n(\sqrt{e}-1)}\left[\sum_{x_{j} \in X_{1}}\left\{\begin{array}{c}
\left\{\begin{array}{c}
\left(\frac{\mu_{B}\left(x_{j}\right)+\mu_{C}\left(x_{j}\right)}{2}\right) \exp \left(\frac{2-\mu_{B}\left(x_{j}\right)-\mu_{C}\left(x_{j}\right)}{2}\right) \\
+\left(\frac{2-\mu_{B}\left(x_{j}\right)-\mu_{C}\left(x_{j}\right)}{2}\right) \exp \left(\frac{\mu_{B}\left(x_{j}\right)+\mu_{C}\left(x_{j}\right)}{2}\right)
\end{array}\right\} \\
-\left\{\begin{array}{l}
\left(\mu_{B}\left(x_{j}\right) \exp \left(1-\mu_{B}\left(x_{j}\right)\right)+\left(1-\mu_{B}\left(x_{j}\right)\right) \exp \left(\mu_{B}\left(x_{j}\right)\right)\right) \\
+\left(\mu_{C}\left(x_{j}\right) \exp \left(1-\mu_{C}\left(x_{j}\right)\right)+\left(1-\mu_{C}\left(x_{j}\right)\right) \exp \left(\mu_{C}\left(x_{j}\right)\right)\right) \\
2
\end{array}\right\}
\end{array}\right\}\right. \\
& +\sum_{x_{j} \in X_{2}}\left\{\begin{array}{c}
\left\{\begin{array}{c}
\left(\frac{\mu_{A}\left(x_{j}\right)+\mu_{C}\left(x_{j}\right)}{2}\right) \exp \left(\frac{2-\mu_{A}\left(x_{j}\right)-\mu_{C}\left(x_{j}\right)}{2}\right) \\
+\left(\frac{2-\mu_{A}\left(x_{j}\right)-\mu_{C}\left(x_{j}\right)}{2}\right) \exp \left(\frac{\mu_{A}\left(x_{j}\right)+\mu_{C}\left(x_{j}\right)}{2}\right)
\end{array}\right\} \\
-\left\{\begin{array}{c}
\left(\mu_{A}\left(x_{j}\right) \exp \left(1-\mu_{A}\left(x_{j}\right)\right)+\left(1-\mu_{A}\left(x_{j}\right)\right) \exp \left(\mu_{A}\left(x_{j}\right)\right)\right) \\
+\left(\mu_{C}\left(x_{j}\right) \exp \left(1-\mu_{C}\left(x_{j}\right)\right)+\left(1-\mu_{C}\left(x_{j}\right)\right) \exp \left(\mu_{C}\left(x_{j}\right)\right)\right) \\
2
\end{array}\right\}
\end{array}\right\} .
\end{aligned}
$$


6. Equation (24) is in error, the correct form is as follows:

$$
\begin{gathered}
=\frac{1}{n(\sqrt{e}-1)}\left[\sum_{x_{j} \in X_{1}}\left\{\begin{array}{c}
\left\{\begin{array}{c}
\left(\frac{\mu_{B}\left(x_{j}\right)+\mu_{C}\left(x_{j}\right)}{2}\right) \exp \left(\frac{2-\mu_{B}\left(x_{j}\right)-\mu_{C}\left(x_{j}\right)}{2}\right) \\
+\left(\frac{2-\mu_{B}\left(x_{j}\right)-\mu_{C}\left(x_{j}\right)}{2}\right) \exp \left(\frac{\mu_{B}\left(x_{j}\right)+\mu_{C}\left(x_{j}\right)}{2}\right)
\end{array}\right\} \\
-\left\{\begin{array}{c}
\left(\mu_{B}\left(x_{j}\right) \exp \left(1-\mu_{B}\left(x_{j}\right)\right)+\left(1-\mu_{B}\left(x_{j}\right)\right) \exp \left(\mu_{B}\left(x_{j}\right)\right)\right) \\
+\left(\mu_{C}\left(x_{j}\right) \exp \left(1-\mu_{C}\left(x_{j}\right)\right)+\left(1-\mu_{C}\left(x_{j}\right)\right) \exp \left(\mu_{C}\left(x_{j}\right)\right)\right) \\
2
\end{array}\right.
\end{array}\right\}\right) \\
+\sum_{x_{j} \in X_{2}}\left\{\begin{array}{c}
\left\{\begin{array}{c}
\left(\frac{\mu_{A}\left(x_{j}\right)+\mu_{C}\left(x_{j}\right)}{2}\right) \exp \left(\frac{2-\mu_{A}\left(x_{j}\right)-\mu_{C}\left(x_{j}\right)}{2}\right) \\
+\left(\frac{2-\mu_{A}\left(x_{j}\right)-\mu_{C}\left(x_{j}\right)}{2}\right) \exp \left(\frac{\mu_{A}\left(x_{j}\right)+\mu_{C}\left(x_{j}\right)}{2}\right)
\end{array}\right\} \\
\left.-\left\{\begin{array}{c}
\left(\mu_{A}\left(x_{j}\right) \exp \left(1-\mu_{A}\left(x_{j}\right)\right)+\left(1-\mu_{A}\left(x_{j}\right)\right) \exp \left(\mu_{A}\left(x_{j}\right)\right)\right) \\
+\left(\mu_{C}\left(x_{j}\right) \exp \left(1-\mu_{C}\left(x_{j}\right)\right)+\left(1-\mu_{C}\left(x_{j}\right)\right) \exp \left(\mu_{C}\left(x_{j}\right)\right)\right) \\
2
\end{array}\right\}\right) .
\end{array}\right] .
\end{gathered}
$$

7. In Eqs. (28) and (29), “ $\frac{1}{n}$, should be replaced by ' $\frac{1}{n(\sqrt{e}-1)}$ '.

8. The correct forms of Tables 1,2 and 3 are as follows:

9. In Step 1 of numerical example, first expression for $M^{+}$is correct, and the error is the next line. It gives in fact $M^{-}$. Thus, Step 1 in corrected form is to be:
Step 1 Obtaining the $M^{+}$and $M^{-}$given by $M^{+}=\left\{\left\langle C_{1}, 0.8\right\rangle,\left\langle C_{2}, 0.7\right\rangle,\left\langle C_{3}, 0.7\right\rangle,\left\langle C_{4}, 0.6\right\rangle\right\}$,
$M^{-}=\left\{\left\langle C_{1}, 0.5\right\rangle,\left\langle C_{2}, 0.4\right\rangle,\left\langle C_{3}, 0.3\right\rangle,\left\langle C_{4}, 0.2\right\rangle\right\}$.

Table 1 Values of $\operatorname{FJED}\left(M^{+} \mid M_{i}\right)$

\begin{tabular}{lllll}
\hline $\operatorname{FJED}\left(M^{+} \mid M_{1}\right)$ & $\operatorname{FJED}\left(M^{+} \mid M_{2}\right)$ & $\operatorname{FJED}\left(M^{+} \mid M_{3}\right)$ & $\operatorname{FJED}\left(M^{+} \mid M_{4}\right)$ & $\operatorname{FJED}\left(M^{+} \mid M_{5}\right)$ \\
\hline 0.1018 & 0.0121 & 0.0293 & 0.0797 & 0.0340 \\
\hline
\end{tabular}

Table 2 Values of $\operatorname{FJED}\left(M^{-} \mid M_{i}\right)$

\begin{tabular}{lllll}
\hline $\operatorname{FJED}\left(M^{-} \mid M_{1}\right)$ & $\operatorname{FJED}\left(M^{-} \mid M_{2}\right)$ & $\operatorname{FJED}\left(M^{-} \mid M_{3}\right)$ & $\operatorname{FJED}\left(M^{-} \mid M_{4}\right)$ & $\operatorname{FJED}\left(M^{-} \mid M_{5}\right)$ \\
\hline 0.0095 & 0.0797 & 0.0533 & 0.0317 & 0.0629 \\
\hline
\end{tabular}

Table 3 Values of $\operatorname{FJED}\left(M_{i}\right)$

\begin{tabular}{lllll}
\hline $\operatorname{FJED}\left(M_{1}\right)$ & $\operatorname{FJED}\left(M_{2}\right)$ & $\operatorname{FJED}\left(M_{3}\right)$ & $\operatorname{FJED}\left(M_{4}\right)$ & $\operatorname{FJED}\left(M_{5}\right)$ \\
\hline 0.9146 & 0.1318 & 0.3547 & 0.7154 & 0.3509 \\
\hline
\end{tabular}

